\title{
DOS NUEVOS PUNTOS FOSILÍFEROS CON MACROMAMÍFEROS DEL ARAGONIENSE DE LA CUENCA DE MONTALBÁN (TERUEL, ESPAÑA)
}

\author{
Ana Victoria MAZO, Manuel Jesús SALESA \\ e Israel Miguel SÁNCHEZ
}

Departamento de Paleobiología, Museo Nacional de Ciencias Naturales-CSIC, C/ José Gutiérrez Abascal, 2, 28006 Madrid, España.

\begin{abstract}
Mazo, A.V., Salesa, M.J. y Sánchez, I.M. 2002. Dos nuevos puntos fosilíferos con macromamíferos del Aragoniense de la cuenca de Montalbán (Teruel, España). [Two new macromammal fossil sites from the Aragonian of the Montalbán basin (Teruel, Spain).] Revista Española de Paleontología, 17(2), 269-283. ISSN 0213-6937.
\end{abstract}

\begin{abstract}
In the present paper two new fossil mammal assemblages (Bañon and Balsete sites, Teruel province, Spain) are discussed. The samples consist mainly of a great number of bone fragments, so in most cases the taxonomic identifications of the macrofauna have been made only at family level. Even so, two species have been identified allowing the dating of the sites: Hispanotherium matritense Prado, 1864 (at Balsete) which characterizes the biozone D, and Heteroprox moralesi Azanza, 2000 (at Bañón) which is present in the zone MN 6 of the Aragonian. The new sites have provided not much fossil material, but are of high interest in the study of the paleobiogeography of some species, such as the cervid Heteroprox moralesi, because this is the first recording of this cervid outside the Madrid basin.
\end{abstract}

Keywords: Anchitherium, Hispanotherium, Gomphotherium, Heteroprox, Suidae, Carnivora, Aragonian, middle Miocene.

\section{RESUMEN}

En el presente trabajo se estudia la fauna de macromamíferos de los nuevos yacimientos de Balsete (Cosa) y Bañón, ambos en la provincia de Teruel. El material estudiado está, en la mayoría de los casos, muy fragmentado, de manera que las identificaciones taxonómicas sólo han podido realizarse a nivel de familia; sin embargo se ha podido identificar dos especies que han permitido datar los yacimientos: Hispanotherium matritense Prado, 1864 (en Balsete), que caracteriza la biozona D y Heteroprox moralesi Azanza, 2000 (en Bañón) cérvido típico de la zona MN 6 del Aragoniense. Los nuevos yacimientos no han proporcionado demasiado material, pero éste tiene un alto interés en el estudio de la paleobiogeografía de algunas de las especies presentes, como el cérvido Heteroprox moralesi, del cual Bañón constituye el primer registro fuera de la cuenca de Madrid.

Palabras clave: Anchitherium, Hispanotherium, Gomphotherium, Heteroprox, Suidae, Carnivora, Aragoniense, Mioceno medio.

\section{INTRODUCCIÓN}

La cuenca terciaria de Calatayud-Montalbán se localiza en el NE de la Península Ibérica, entre las cuencas de Almazán y del Ebro, formando parte del sistema de fosas y subfosas tectónicas de carácter intramontañoso enclavadas en la Cordillera Ibérica (Fig. 1). La cuenca terciaria de Calatayud-Montalbán consta de dos subcuencas diferentes que presentan secuencias similares de relleno, aunque con distinta crono- estratigrafía, separadas por el umbral paleozoico de Daroca. El sector septentrional se corresponde con la cuenca de Calatayud, mientras que el sector meridional lo hace con la cuenca de Montalbán (Fig. 2). En la cuenca de Calatayud se reconocen tradicionalmente en el Neógeno tres unidades sedimentarias mayores denominadas Unidad Inferior, Intermedia y Superior separadas por dos rupturas sedimentarias principales (Sanz-Rubio, 1999). La correlación de las tres unidades neógenas de la cuenca de Calatayud con las de la vecina 


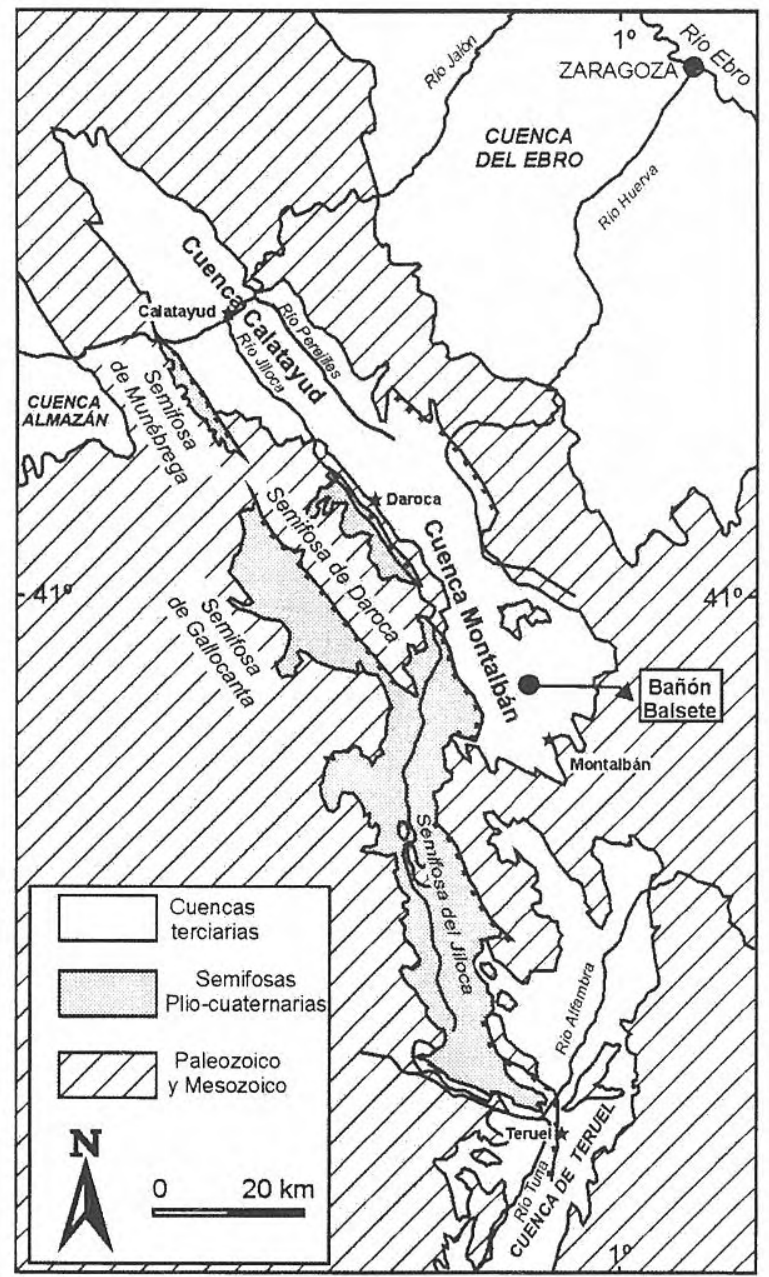

Figura 1. Localización de los puntos fosilíferos de Balsete y Bañón dentro de la cuenca de CalatayudMontalbán.

Situation of Bañon and Balsete fossil sites within the Calatayud-Montalbán Basin.

cuenca de Montalbán ofrece pequeñas diferencias cronoestratigráficas. Mientras que en la cuenca de Calatayud las tres unidades mayores fueron definidas en función de dos rupturas sedimentarias observables regionalmente, en los sectores centrales de la cuenca de Montalbán (UTS 5, Casas et al., 2000) se aprecia una evolución sedimentaria muy similar para las unidades neógenas, aunque las dos rupturas sedimentarias en el sector septentrional de Calatayud no son evidentes, debido a una ausencia de registro por erosión y también a la carencia de un adecuado control bioestratigráfico.

La cuenca de Montalbán constituye una depresión originada en régimen compresivo que ha sido rellenada por sedimentos sin-tectónicos desde el Eoceno hasta el Mioceno inferior, y que comprende sedimentos lacustres y aluviales que alcanzan una potencia máxima de unos $2.000 \mathrm{~m}$ (Casas et al., 2000). La cuenca posee una distribución concéntrica de facies, donde depósitos groseros clásticos en los bordes pasan progresivamente a carbonatos y evaporitas hacia el centro de la cuenca. Las facies centrales de las unidades neógenas de la cuenca de

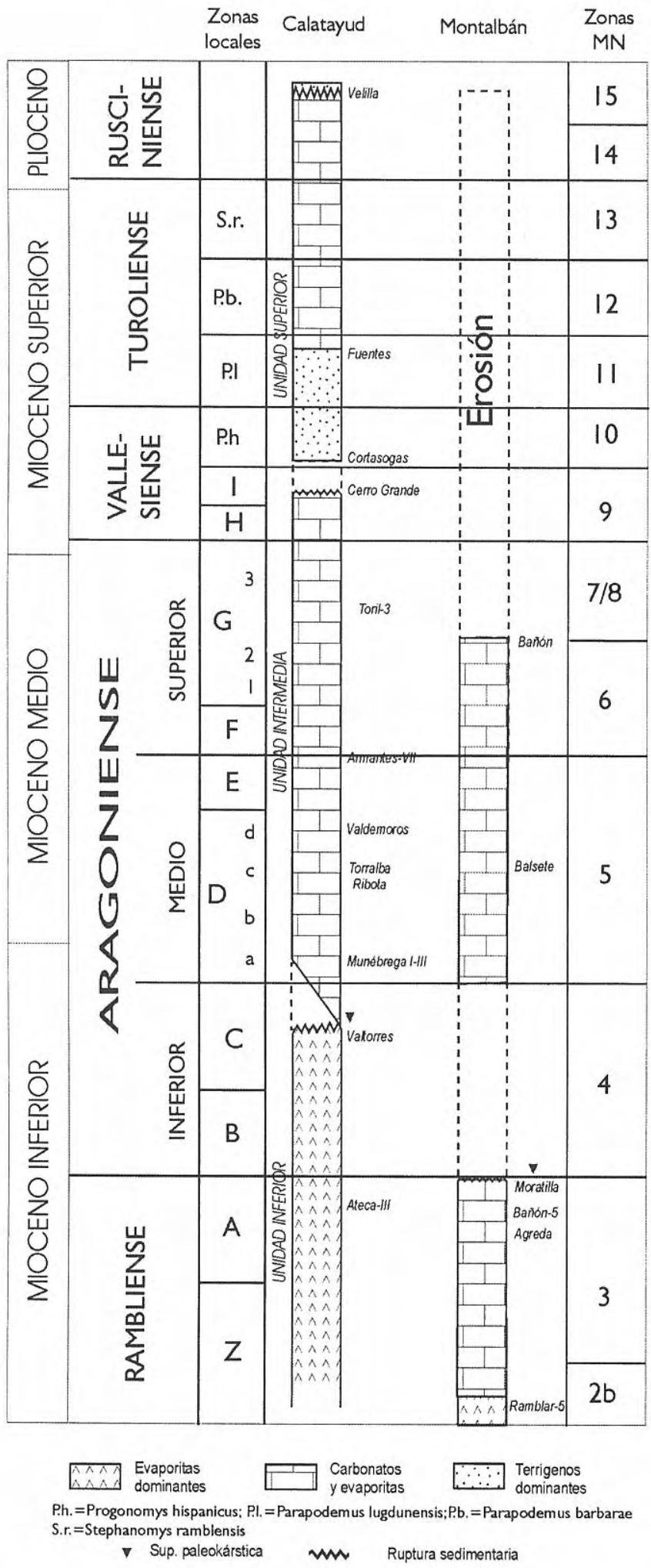

Figura 2. Localización estratigráfica de los yacimientos de Bañón y Balsete (modificado de Sanz-Rubio, 1999).

Stratigraphic situation of Bañón and Balsete sites (modified from Sanz-Rubio, 1999).

Montalbán corresponden a evaporitas y carbonatos que afloran en un área confinada aproximadamente por las 
localidades de Calamocha, Bañón, Cosa, Torrelosnegros y Navarrete del Río.

Desde 1975 se estudian con especial interés las relaciones de la cuenca de Montalbán con la vecina cuenca de Calatayud (sector septentrional de la cuenca de Calatayud-Montalbán). En ambas la secuencia sedimentaria parece ser similar, aunque teniendo en cuenta que el registro de una y otra contiene sedimentos que no son de las mismas edades. Prospecciones geológicas y paleontológicas llevadas a cabo en los últimos años en las zonas de Bañón, Navarrete del Río y Daroca-Villafeliche han dado lugar a trabajos decisivos para el conocimiento del área (Daams y Freudenthal, 1988; Daams y van der Meulen, 1992). En Daams y Freudenthal (op. cit.) se citan 3 localidades denominadas Bañón 2, Bañón 5 y Bañón 11, que bioestratigráficamente sitúan en el Rambliense superior, zona A, de la biozonación local.

Recientemente, y en el contexto del Proyecto de la D. G. E. S. PB-98-0503 titulado "Cambios mayores y ciclicidad sedimentaria en las secuencias neógenas de precipitación salina de las cuencas de Montalbán y Calatayud: geomarcadores de la evolución paleoclimática en cuencas terciarias ibéricas", durante la realización de unos trabajos de cartografía geológica, se localizaron casualmente dos nuevos puntos fosilíferos de los cuales se recuperaron restos de macro y microfauna. En este trabajo estudiaremos y situaremos en el contexto bioestratigráfico de la zona los yacimientos que denominaremos Bañón, situado en el Km 128 de la carretera N- 211 y Balsete, situado en el Km 134 de la misma carretera, próximo al pueblo de Cosa. Bañón ha proporcionado fósiles de macro y microvertebrados; Balsete, por el momento, no ha sido muestreado para la posible obtención de microfauna.

En la actualidad se lleva a cabo un trabajo general en el que se recogen todos los estudios interdisciplinares realizados y los resultados obtenidos.

\section{MATERIAL Y MÉTODO}

Los fósiles recuperados están depositados temporalmente en el Museo Nacional de Ciencias Naturales de Madrid para su estudio y restauración, entregándose, cuando ello finalice, a la Diputación General de Aragón. El estado de conservación es malo, ya que la mayoría de los restos consisten en fragmentos de pequeño tamaño que no permiten identificaciones taxonómicas precisas. Varias de las piezas significativas estaban igualmente fragmentadas pero han podido reconstruirse.

El estudio comparativo se ha basado especialmente en las revisiones específicas que existen sobre cada grupo: para los cérvidos, Azanza (2000); para los rinocerontes, Cerdeño (1989); en el caso de los mastodontes, Mazo (1997); para el género Anchitherium, el trabajo de Sánchez et al. (1998); y para los carnívoros, Ginsburg (1961) y la comparación con material actual.

Las abreviaturas que figuran en el texto y tablas son las siguientes: BÑ, Bañón; BS, Balsete; A, anchura; L, longitud;
H, altura; DAP, diámetro antero-posterior; DT, diámetro transverso; FC 1, primera falange central; FC 2, segunda falange central; MC III, metacarpiano $3^{\circ}$; MT III, metatarsiano $3^{\circ}$; M, longitud mesiodistal; B, anchura bucolingual; M/B, índice entre ambas medidas. Todas las medidas se expresan en milímetros. Las medidas de los elementos postcraneales del género Anchitherium se han tomado siguiendo las recomendaciones de Eisenmann et al. (1988), adaptadas por Sánchez et al. (1998) para este género.

Los análisis estadísiticos se realizaron utilizando los programas STATISTICA 4.1 para Mac y SPSS 9.0 para PC.

\section{PALEONTOLOGÍA SISTEMÁTICA}

\section{LOCALIDAD: BALSETE, COSA (TERUEL)}

Del nuevo yacimiento que hemos denominado Balsete, sólo se han recuperado restos asignables a un mastodonte (probablemente Gomphotherium angustidens) y a un rinoceronte identificado como Hispanotherium matritense. Por el momento este yacimiento no ha sido muestreado para la obtención de microfauna.

\section{ORDEN PROBOSCIDEA Illiger, 1811} Familia Gomphotheriidae Hay, 1922

\section{Gomphotheriidae indet.}

\section{BS-04 a BS-14: varios fragmentos de diferentes tamaños, pertenecientes a un incisivo superior}

El estado de conservación es muy malo: ninguno de los fragmentos conserva la sección completa, sino que ha fosilizado sólo una mitad rota longitudinalmente. No se ven restos de banda de esmalte, aunque teniendo en cuenta la conservación no puede excluirse que existiera. El fragmento de incisivo no proporciona ninguna otra información.

\section{ORDEN PERISSODACTYLA Owen, 1848}

Familia Rhinocerotidae Owen, 1845

Subfamilia Elasmotheriinae Dollo, 1885

Género Hispanotherium Crusafont y Villalta, 1947

\section{Hispanotherium matritense Prado, 1864}

Figs. 3a-b

\section{Fragmentos de $\mathbf{m} 3$ derecho (BS-01 y BS-02)}

El resto conservado es la región posterior de un $\mathrm{m} 3$, hipsodonto y con desgaste medio, cuyo valle posterior está lleno de cemento; el cíngulo distal está muy poco marcado.

La presencia de cemento y la altura de la corona permiten identificar este molar como perteneciente a Hispanotherium matritense; este rinoceronte de pequeña talla y con un cuerno nasal, fue descrito por primera vez por Prado (1864) en el yacimiento de Puente de Toledo (Madrid). Se ha determinado también en otros yacimientos madrileños, como Estación Imperial, Paseo de las Acacias y Paseo de la Esperanza (Soria et al., 2000). Se conoce igualmente en Torrijos (Toledo), Córcoles (Guadalajara) (Alférez et al., 1999), La Retama (Cuenca) (Morales et al., 


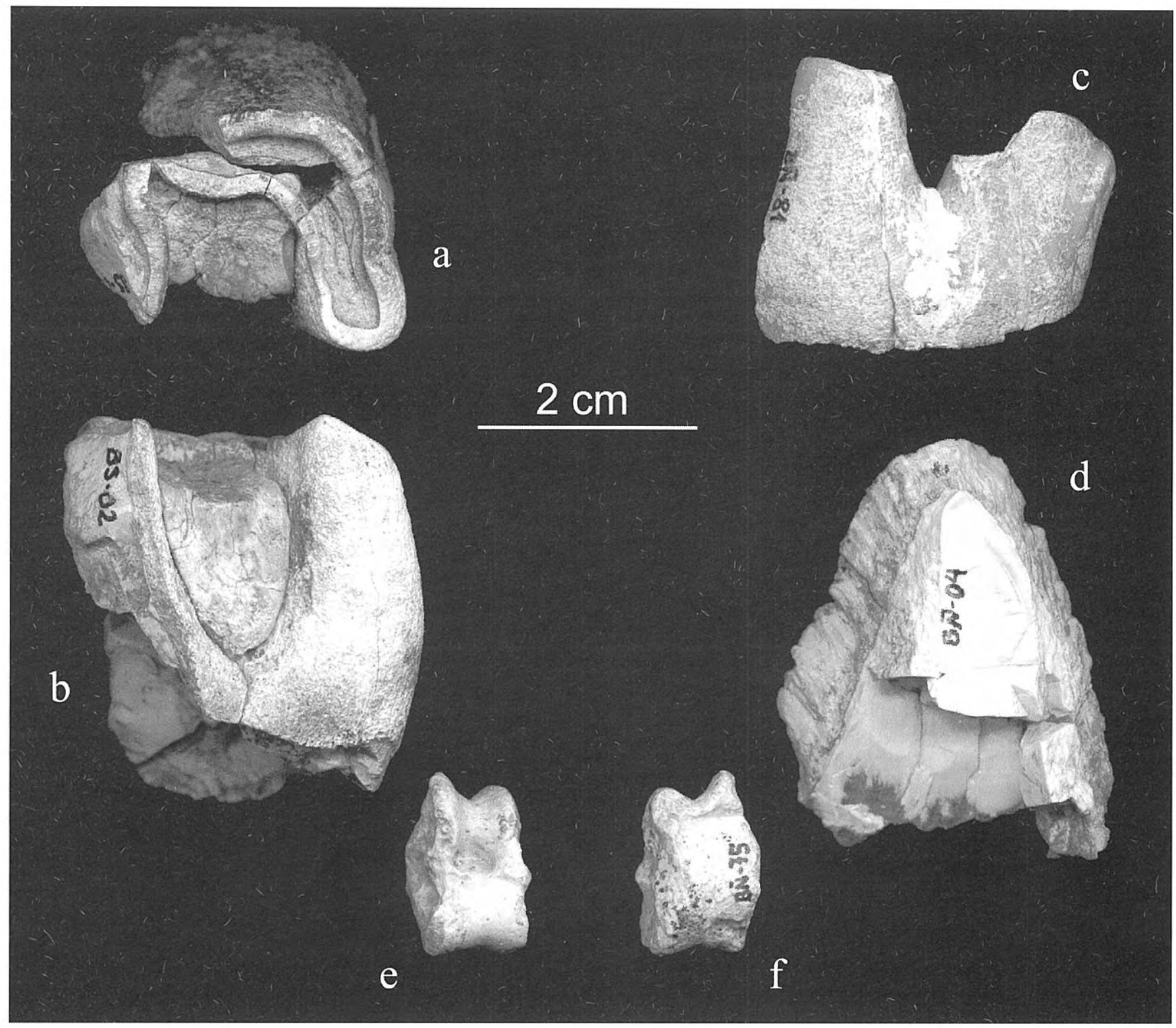

Figura 3. a-b, BS-02: fragmento de diente yugal inferior de Hispanotherium matritense de Balsete; a, vista oclusal; b, vista lingual. c, BÑ-81: fragmento de diente yugal de Rhinocerotidae indet. de Bañón, vista bucal. d, BÑ-04: fragmento de diente yugal de Gomphoteriidae indet. de Bañón. e-f, BÑ-75: astrágalo derecho de Pecora indet. de Bañón; e, vista dorsal; f, vista ventral.

$a-b, B S-02:$ lower jugal tooth fragment of Hispanotherium matritense from Balsete; a, occlusal view; $b$, lingual view. $c$, BÑ-81: jugal tooth fragment of Rhinocerotidae indet. from Bañon, bucal view. d, BÑ-04: jugal tooth fragment of Gomphoteriidae indet. from Bañón. e-f, BÑ-75: right astragalus of Pecora indet. from Bañón; e, dorsal view; $f$, ventral view.

1993; Soria et al., 2000), Plasencia (Cáceres) (HernándezPacheco y Crusafont, 1960), y en los yacimientos zaragozanos de Torralba de Ribota V, Valdemoros 1, Munébrega I (Cerdeño, 1989) y Tarazona de Aragón (Astibia, 1987; Astibia y Mazo, 1988).

Aunque inicialmente se tuvo por una forma endémica de la Península Ibérica, está presente también en Francia (Ginsburg y Telles Antunes, 1979), Turquía (Heissig, 1974, 1976) y Mongolia. En nuestra Península caracteriza la biozona D del Aragoniense medio, mientras que en Turquía se conoce desde el Aragoniense medio (MN 5) hasta el final del Aragoniense superior.

\section{LOCALIDAD: BAÑON (TERUEL)}

ORDEN PROBOSCIDEA Owen, 1811

Familia Gomphotheriidae Hay, 1922 Gomphotheriidae indet.

\section{Fig. 3d}

Fragmento de cónulo de molar (BÑ-04) y fragmento de incisivo (BÑ -21)

El grosor del esmalte y su aspecto en norma lateral identifica el cónulo como perteneciente a un mastodonte bunodonto. El fragmento de incisivo no aporta más datos. 


\begin{tabular}{|cccccc|}
\hline Sigla & Pieza & Mesiodistal & Bucolingual & Indice M/B & Altura \\
\hline BÑ-73 & $\mathrm{p} 4$ & 23,75 & 18,17 & 1,31 & 12,42 \\
BÑ-02 & $\mathrm{P} 4$ & $25,1,3$ & 30,11 & 0,83 & - \\
\hline
\end{tabular}

Tabla 1. Medidas de la dentición de Anchitherium sp. de Bañón.

Dental measurements of Anchitherium sp. from Bañón.

Teniendo en cuenta la determinación de la fauna acompañante, es más que probable que estos restos pertenezcan a Gomphotherium angustidens (Cuvier, 1817), pero siendo rigurosos sólo debe identificarse a nivel de familia. A pesar del número de fragmentos que puede generar la rotura de un incisivo o de un molar de mastodonte y a la gran cantidad de fragmentos y esquirlas recogidos en Bañón, no se ha recuperado ningún otro resto de proboscídeo.

\section{ORDEN PERISSODACTYLA Owen, 1848 \\ Familia Rhinocerotidae Owen, 1845}

Rhinocerotidae indet.

Fig. 3c

\section{Fragmentos de diente yugal (BÑN-81)}

El fragmento de mayor tamaño es parte de la muralla de un premolar cuyo aspecto y esmalte crenulado identifican como perteneciente a un Rhinocerotidae indet., sin que sea posible su identificación a nivel genérico.

Familia Equidae Gray, 1821

Subfamilia Anchitheriinae Leidy, 1869

Género Anchitherium Meyer, 1834

\section{Anchitherium sp.}

Figs. 4 y 5

Fragmento de maxilar derecho con P3 y P4 (BÑ-02) (Fig. 4a) (medidas en Tabla 1)

Se describe solamente el P4, ya que el P3 está muy fragmentado. El ectolofo está ligeramente inclinado en sentido distal; el mesostilo presenta algo más de desarrollo bucolingual que el parastilo, y el metastilo está muy poco desarrollado. El cíngulo bucal aparece muy marcado; el cíngulo lingual está bien desarrollado a nivel del protocono y apenas a nivel del hipocono. Los bordes linguales del protocono e hipocono se encuentran al mismo nivel. El hipostilo es subtriangular, con una foseta bucal, sin cresta de unión con el metalofo. Existe un pequeño pliegue prefoseta. El valle central carece de estilos en su entrada.

p4 izquierdo (BÑ-73) (Fig. 4b) (medidas en Tabla 1)

La pieza no presenta desgaste y carece de raíces, de lo que se deduce que no llegó a hacer erupción. El protolófidoparalófido presenta una anchura algo menor que el metalófido-hipolófido. El paracónido es notablemente más bajo que el resto de cúspides. El hipoconúlido está bien desarrollado, aunque es algo menor que el entocónido. El metacónido es ligeramente más alto que el metastílido y

\begin{tabular}{|ccccccc|}
\hline Sigla & $\mathbf{1}$ & $\mathbf{2}$ & $\mathbf{3}$ & $\mathbf{4}$ & $\mathbf{5}$ & $\mathbf{6}$ \\
\hline $\mathrm{BN}-03$ & - & - & 60,12 & 40,36 & - & - \\
\hline
\end{tabular}

Tabla 2. Medidas de la escápula de Anchitherium sp. de Bañón.

Scapula measurements of Anchitherium sp. from Bañón.

aparece bien separado de éste. Los valles mesial y distal presentan una longitud similar. El cíngulo bucal está presente pero reducido, mientras que el cíngulo lingual está ausente. Por el contrario, los cíngulos mesial y distal están bien marcados.

\section{Fragmento de maxilar izquierdo con M1 y M2 (BÑ-50)} (Fig. 4c)

El M1 se encuentra muy deteriorado y el M2 algo mejor conservado; no han podido ser medidos debido a la existencia de varias roturas con posterior desplazamiento. El ectolofo aparece ligeramente inclinado en sentido distal; el mesostilo presenta algo más de desarrollo bucolingual que el parastilo en el M1 e igual desarrollo en el M2; el metastilo está muy poco desarrollado. El cíngulo bucal aparece muy marcado; el cíngulo lingual está bien desarrollado a nivel del protocono y muy poco a nivel del hipocono. El borde lingual del protocono sobresale ligeramente del nivel del hipocono. El hipostilo es subtriangular, con una foseta bucal, y carece de cresta de unión con el metalofo. En ambas piezas existe un diminuto pliegue prefoseta. El valle central carece de estilos en su entrada.

\section{Escápula (B̃̃-03) (medidas en Tabla 2)}

El único ejemplar se corresponde con la cavidad glenoidea y los alrededores, incluyendo el tubérculo supraglenoideo, de una escápula izquierda. No se conserva nada más que la primera porción del cuello de la escápula, y el comienzo de las fosas supraespinosa e infraespinosa. La cavidad glenoidea es elíptica y cóncava, presentando los bordes bastante erosionados. El tubérculo supraglenoideo es grande y bien diferenciado, y está claramente separado de la cavidad glenoidea. Presenta un proceso coracoides pequeño y de relieve bien definido.

\section{Navicular (BÑ-17)}

Es un fragmento extremadamente reducido de la parte anterior de un navicular. Aunque permite su identificación, no se puede describir nada de este resto.

Escafoides derecho (Bत̃-82) (Figura 5a-b; medidas en Tabla 3)

\begin{tabular}{|cccc|}
\hline Sigla & Longitud & Anchura & Altura \\
\hline BÑ-82 & 31,8 & 20,65 & 24,09 \\
\hline
\end{tabular}

Tabla 3. Medidas del escafoides de Anchitherium sp. de Bañón.

Scaphoid measurements of Anchitherium sp. from Bañón. 


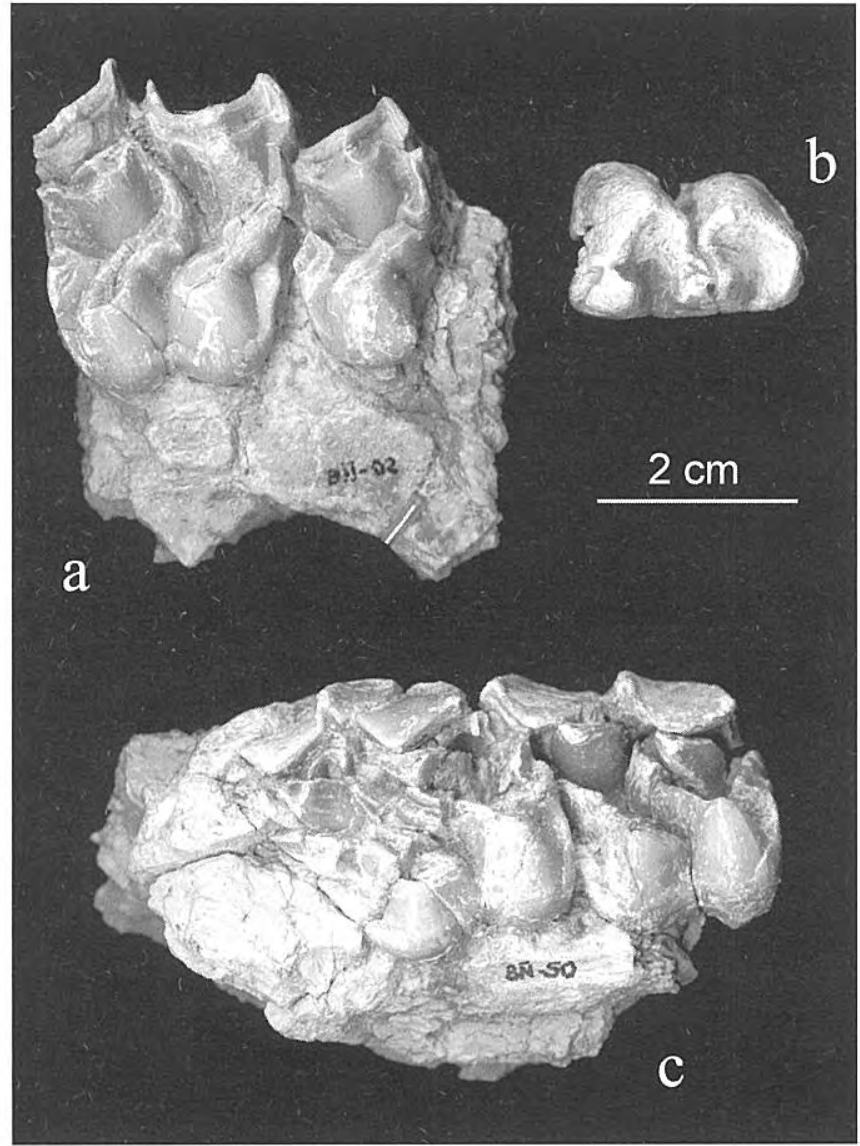

Figura 4. a, BÑ-02: fragmento de maxilar derecho con P3 y P4 de Anchitherium sp. de Bañón, vista oclusal. b, BÑ-73: p4 izquierdo de Anchitherium sp. de Bañón, vista oclusal. c, BÑ-50: fragmento de maxilar izquierdo con M1 y M2 de Anchitherium sp. de Bañón, vista oclusal.

a, BN-02: right maxilla fragment with P3 and P4 of Anchitherium sp. from Bañón, occlusal view. $b, B \tilde{N}-73$ : left $p 4$ of Anchitherium $s p$. from Bañón, occlusal view. c, BÑ-50: left maxilla fragment with $M 1$ and $M 2$ of Anchitherium sp. from Bañón, occlusal view.

La faceta para el radio es subrectangular, anteriormente convexa y posteriormente cóncava. En la cara distal, la faceta para el magno ocupa la mitad anterior de la superficie de articulación. Es una faceta semicircular con un saliente posterior y medial. La faceta para el trapezoide es elíptica y fuertemente cóncava, y se encuentra bastante rota. La faceta proximal para el semilunar está situada anteriormente en la cara lateral del hueso, y es alargada, plana y subrectangular; contacta en toda su longitud con la mitad anterior de la faceta para el radio. La faceta distal para el semilunar está completamente rota.

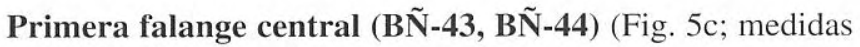
en Tabla 4)

La faceta proximal es subelíptica y cóncava. El surco para la quilla sagital distal del metápodo está poco marcado, y desaparece en la zona central. Las áreas de inserción

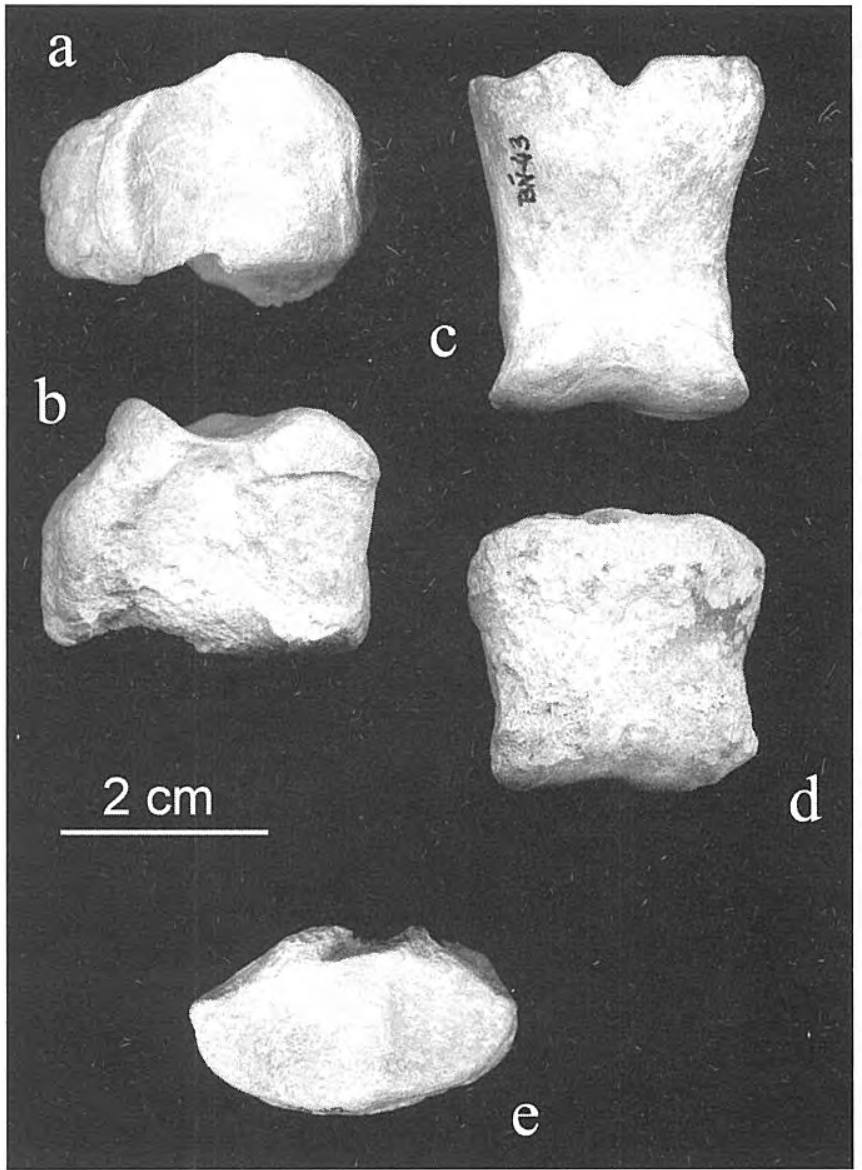

Figura 5. a-b, BÑ-82: escafoides derecho de Anchitherium sp. de Bañón; a, vista proximal; b, vista lateral. c, BÑ-43: $1^{\text {a }}$ falange central de Anchitherium sp. de Bañón, vista dorsal. d, BÑ-74: $2^{a}$ falange central de Anchitherium sp. de Bañón, vista dorsal. e, BÑ-06: fragmento de magnum derecho de Anchitherium sp. de Bañón, vista proximal. $\boldsymbol{a}-\boldsymbol{b}, B \tilde{N}-82$ : right scaphoid of Anchitherium sp. fom Bañón; a, proximal view; $b$, lateral view. $c$, $B \tilde{N}-43$ : first central phalanx of Anchitherium sp. from Bañón, dorsal view. d, BÑ-74: second central phalanx of Anchitherium sp. from Bañon, dorsal view. $e, B \tilde{N}$-06: right magnum fragment of Anchitherium sp. from Bañón, proximal view.

ligamentosa proximo-ventrales están bastante erosionadas, formando un relieve semicircular poco desarrollado. Las dos eminencias (medial y lateral) para la inserción del ligamento plantar, generalmente esféricas y bien desarrolladas, se encuentran en ambos ejemplares erosionadas, aunque la única que se conserva en BÑ-44 está un poco menos dañada por la erosión. Las eminencias para el ligamento colateral

\begin{tabular}{|ccccccccc|}
\hline Sigla & $\mathbf{1}$ & $\mathbf{2}$ & $\mathbf{3}$ & $\mathbf{4}$ & $\mathbf{5}$ & $\mathbf{6}$ & $\mathbf{7}$ & $\mathbf{8}$ \\
\hline BÑ-43 & 34,61 & 31,56 & 22,14 & 28,44 & 20,02 & 22,69 & 25 & 13,9 \\
BÑ-44 & 36,75 & 32,93 & - & 30,12 & 20,98 & - & - & 14,42 \\
\hline
\end{tabular}

Tabla 4. Medidas de la FC1 de Anchitherium sp. de Bañón. FCl measurements of Anchitherium sp. from Bañón. 


\begin{tabular}{|ccccccc|}
\hline Sigla & $\mathbf{1}$ & $\mathbf{2}$ & $\mathbf{3}$ & $\mathbf{4}$ & $\mathbf{5}$ & $\mathbf{6}$ \\
\hline BÑ -74 & 27,37 & 21,68 & 25,53 & 29,81 & 18,03 & 26,23 \\
\hline
\end{tabular}

Tabla 5. Medidas de la FC2 de Anchitherium sp. de Bañón. FC2 measurements of Anchitherium sp. from Bañón.

son pequeñas y poco sobresalientes. La faceta distal tiene forma arriñonada, siendo los cóndilos subiguales en talla.

Segunda falange central (BÑ-74) (Fig. 5d; medidas en Tabla 5)

La faceta proximal es arriñonada y cóncava, y presenta dos fosetas articulares separadas por una cresta ancha y roma. La faceta distal está en bastante mal estado, y es cóncava y recurvada. Se conserva parte de la escotadura ventral para el paso del tendón flexor digital profundo. Toda la cara ventral está erosionada en extremo, por lo que no se observa ninguno de los relieves de inserción ligamentosa.

Magno (B̃̃-06, BÑ-80) (Fig. 5e; medidas en Tabla 6)

Ambos ejemplares son fragmentos de la zona anterior de magnos izquierdos, pero sólo BÑ-80 presenta toda esta zona completa. La superficie articular proximal tiene dos facetas separadas por una cresta. Tanto la faceta para el semilunar como la faceta para el escafoides conservan sólo la parte anterior, que es cóncava. En la cara distal, tampoco se conserva prácticamente nada de la faceta para el MC III. La faceta proximal para el trapezoide es subrectangular y ligeramente cóncava, y la distal plana y semicircular, no contactando entre sí. La proximal conecta en toda su longitud con la faceta para el escafoides, mientras que la distal hace lo propio con la faceta para el MC III. La faceta proximal para el unciforme es rectangular y plana, contactando en toda su longitud con la faceta para el semilunar. La faceta distal para el unciforme es subtriangular y está muy mal conservada. Contacta en ángulo recto con la faceta para el MC III.

\section{Metacarpiano III (BÑ-77)}

Es un fragmento proximal izquierdo muy mal conservado, que comprende parte de la faceta para el magno, y la región anterior de la faceta para el unciforme, sin que se pueda describir nada más.

\section{Metatarsiano III (BÑ-36)}

Es un fragmento muy pequeño de la región proximal de un metatarsiano izquierdo, que conserva una pequeña parte de la faceta de articulación con el ectocuneiforme, y la parte anterior de la faceta para el cuboides.

\begin{tabular}{|cccc|}
\hline Sigla & Longitud Anchura & Altura \\
\hline BÑ-06 & - & - & 15,65 \\
BÑ-80 & - & 30,67 & 17,46 \\
\hline
\end{tabular}

Tabla 6. Medidas del magno de Anchitherium sp. de Bañón.

Magnum measurements of Anchitherium sp. from Bañón.

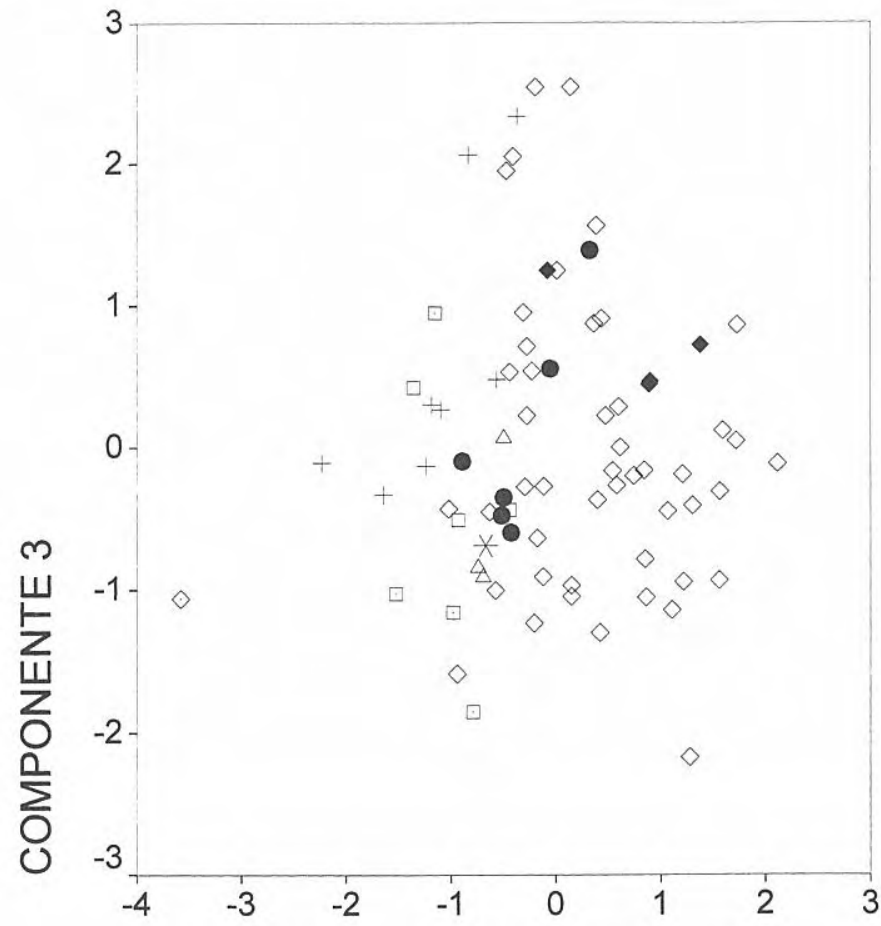

\section{COMPONENTE 1}

\section{LOCALIDADES}

* BAÑón

$\square \quad$ A. DEL OLIVAR

․ IMPERIAL

$\diamond$

$\diamond$
- PARACUELLOS 5

$\triangle \quad$ ACACIAS

$\diamond \quad$ RETAMA

$+\quad$ ALHAMBRA
Figura 6. FCl de Anchitherium sp. Representación gráfica del componente 1 contra el componente 3 . FCl of Anchitherium sp. Scatterplot of Factor 1 and Factor 3.

\section{Discusión}

Hemos utilizado el Análisis de Componentes Principales (PCA) para comparar morfométricamente los restos postcraneales de Anchitherium de Bañón con los de otros yacimientos españoles. Este análisis, debido a la escasez de restos, sólo lo hemos podido llevar a cabo con las primeras y segundas falanges centrales, y con el escafoides. En el caso de las falanges, todos los restos aparecen agrupados en una nube de puntos, sin que se pueda observar ninguna segregación del ejemplar de Bañón respecto del resto (Figs. 6 a 9; Tablas 7 a 10). Sólo cuando analizamos el escafoides podemos observar una agrupación interesante (Fig. 10). El primer componente, que explica un $65,761 \%$ de la varianza total, segrega la muestra por el tamaño, y podemos observar en el gráfico que el escafoides de Bañón se separa como el de mayor 


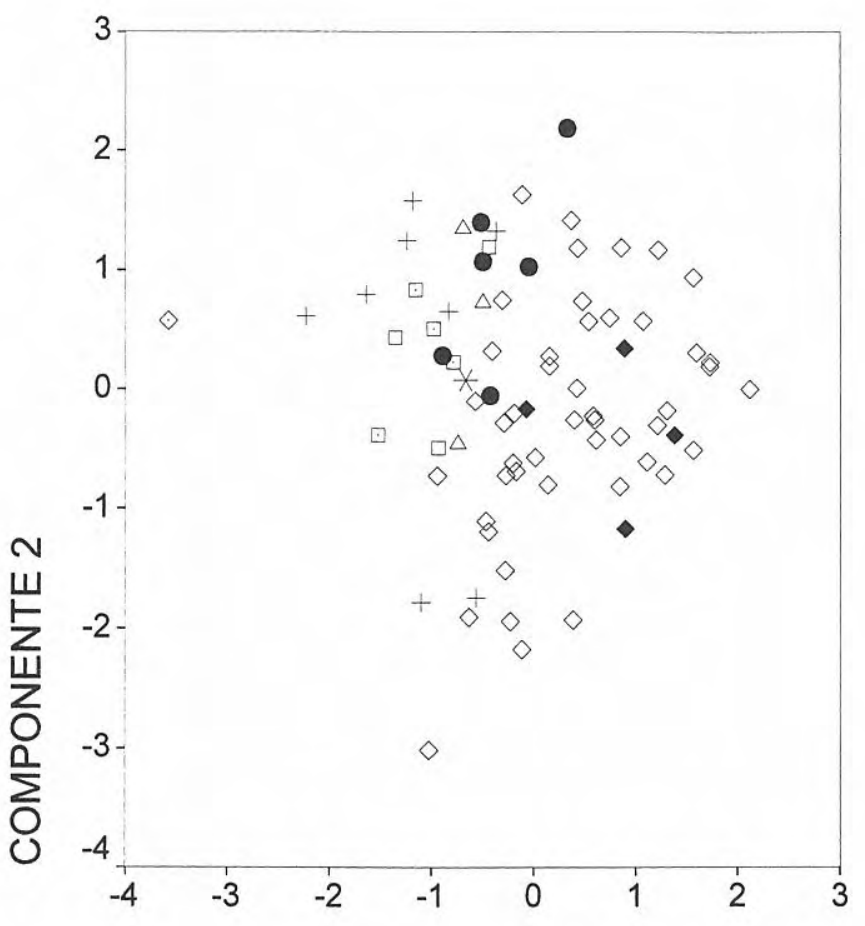

COMPONENTE 1

\section{LOCALIDADES}

\section{* BAÑón}

A. DEL OLIVAR

घ. IMPERIAL

$\diamond \quad$ CÓRCOLES

\section{$\diamond \quad$ EL TERRERO}

Figura 7. FC1 de Anchitherium sp. Representación gráfica del componente 1 contra el componente 2.

FCl of Anchitherium sp. Scatterplot of Factor 1 and Factor 2.

talla de toda la muestra analizada, y que el resto de especies se agrupan bastante bien. El segundo componente, que explica un $27,339 \%$ de la varianza total

\begin{tabular}{|c|ccc|}
\hline Componente & Total & \% Varianza & \% Acumulado \\
\hline 1 & 3,169 & 52,808 & 52,808 \\
2 & 1,817 & 30,28 & 83,088 \\
3 & 0,399 & 6,657 & 89,746 \\
4 & 0,25 & 4,16 & 93,906 \\
5 & 0,233 & 3,886 & 97,791 \\
6 & 0,133 & 2,209 & 100 \\
\hline
\end{tabular}

Tabla 7. PCA FC1 de Anchitherium sp. Varianza total explicada.

PCA FCl of Anchitherium sp. Total Variance Explained.

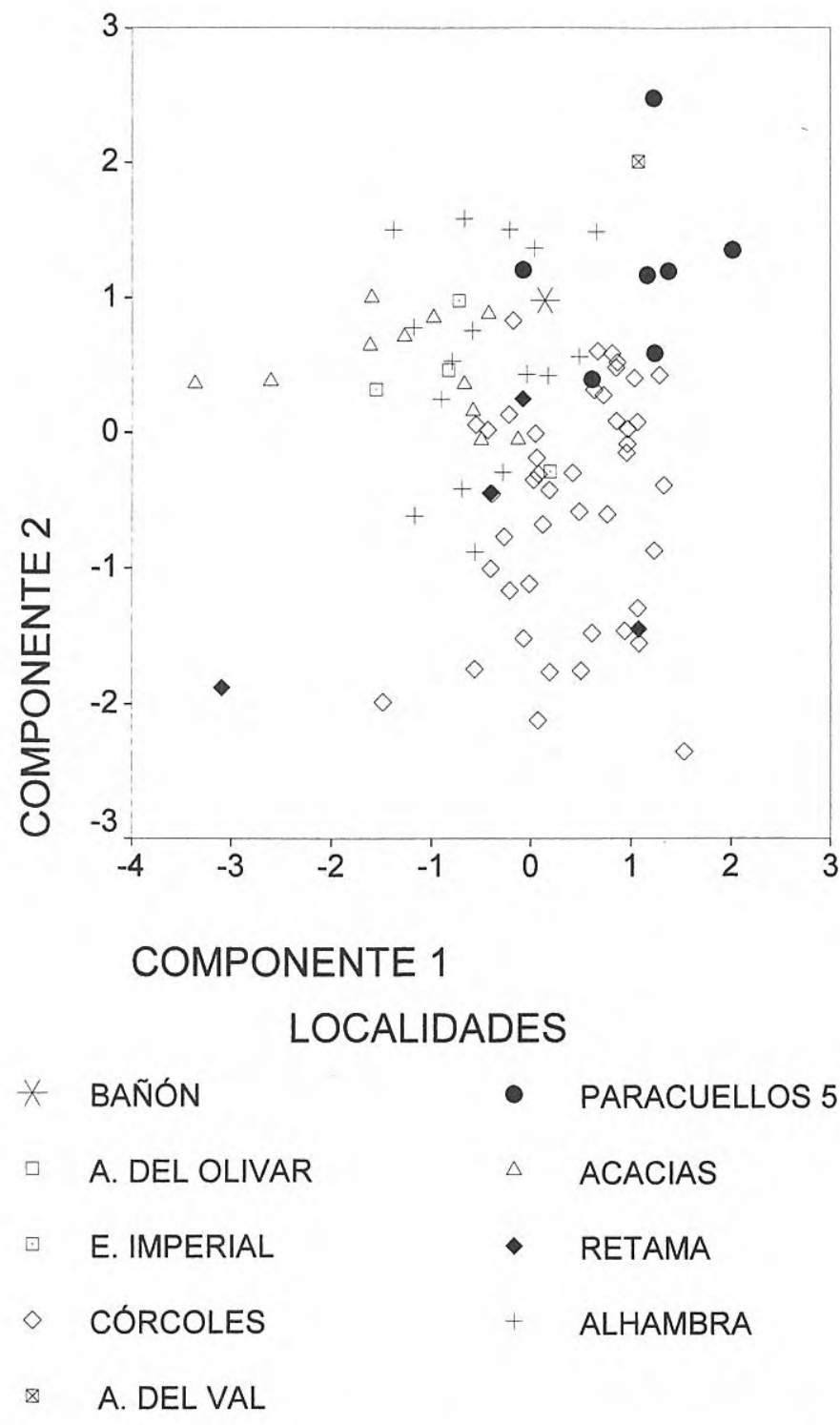

Figura 8. FC2 de Anchitherium sp. Representación gráfica del componente 1 contra el componente 2. FC2 of Anchitherium sp. Scatterplot of Factor 1 and Factor 2.

(93,100\% acumulado) segrega la muestra en función de la morfología del escafoides, separando huesos largos y estrechos de los cortos y más anchos (Tablas 11 y 12). Entre los primeros aparece Bañón, junto con una agrupación muy clara de Alhambra y Arroyo del Olivar (A. cursor), Paracuellos 5 (A. procerum), y parte de la muestra de Puente de Vallecas (A. matritense) y La Retama (A. castellanum), que aparece situada en el centro de las dos nubes. En la nube correspondiente a los escafoides cortos y anchos aparece claramente segregado Córcoles (A. corcolense) (Fig. 10).

En cuanto a los restos dentarios, también son muy escasos y tampoco han permitido una asignación específica. En las figuras 11 y 12 puede observarse que la dentición del anquiterino de Bañón presenta un tamaño similar al de las especies ya conocidas de dentición grande. Se realizaron varios test de la t para analizar esta 


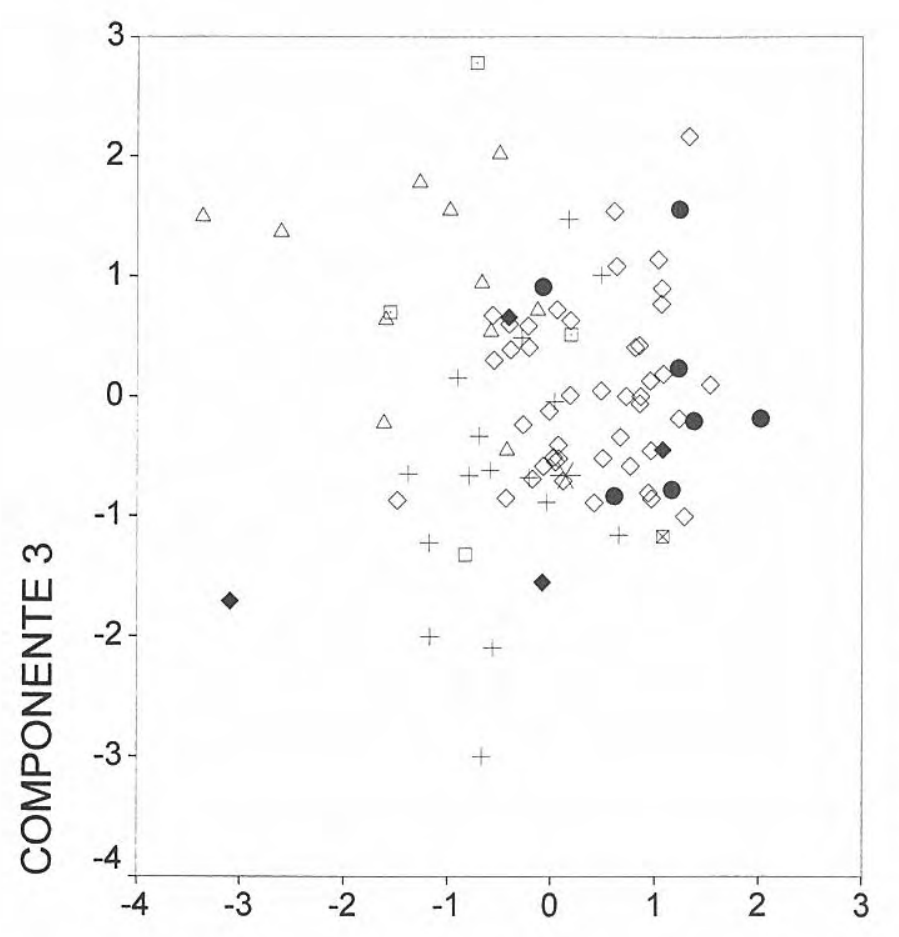

\section{COMPONENTE 1}

\section{LOCALIDADES}

\section{* Bañón}

- A. DEL OLIVAR

$\square$

$\diamond$

$\otimes$

\section{CÓRCOLES}

- Paracuellos 5

$\triangle \quad$ ACACIAS

- retama

+ ALHAMBRA

\section{A. DEL VAL}

Figura 9. FC2 de Anchitherium sp. Representación gráfica del componente 1 contra el componente 3.

FC2 of Anchitherium sp. Scatterplot of Factor 1 and Factor 3.

similitud cuyos resultados muestra la Tabla 13: el p4 tan sólo muestra diferencias significativas respecto a $A$. corcolense, pero el P4 se separa claramente de $A$. castellanum, A. matritense y A. alberdiae. Los restos dentarios de $A$. procerum son tan escasos que no pueden compararse estadísticamente con los de Bañón, aunque el tamaño es muy similar.

Estos resultados no son suficientes para asignar el ejemplar de Bañón a ninguna de las especies conocidas de Anchitherium, aunque el tamaño y morfología del escafoides y de los escasos restos dentarios de los que se dispone probablemente lo acerque a Anchitherium procerum Sánchez, Salesa y Morales, 1998. Asimismo, la escasez y mala conservación de los restos postcraneales hace que no sea posible identificar ninguno de los caracteres morfológicos utilizados para el análisis cladístico de los Anchitheriinae españoles (Sánchez et al., 1998).

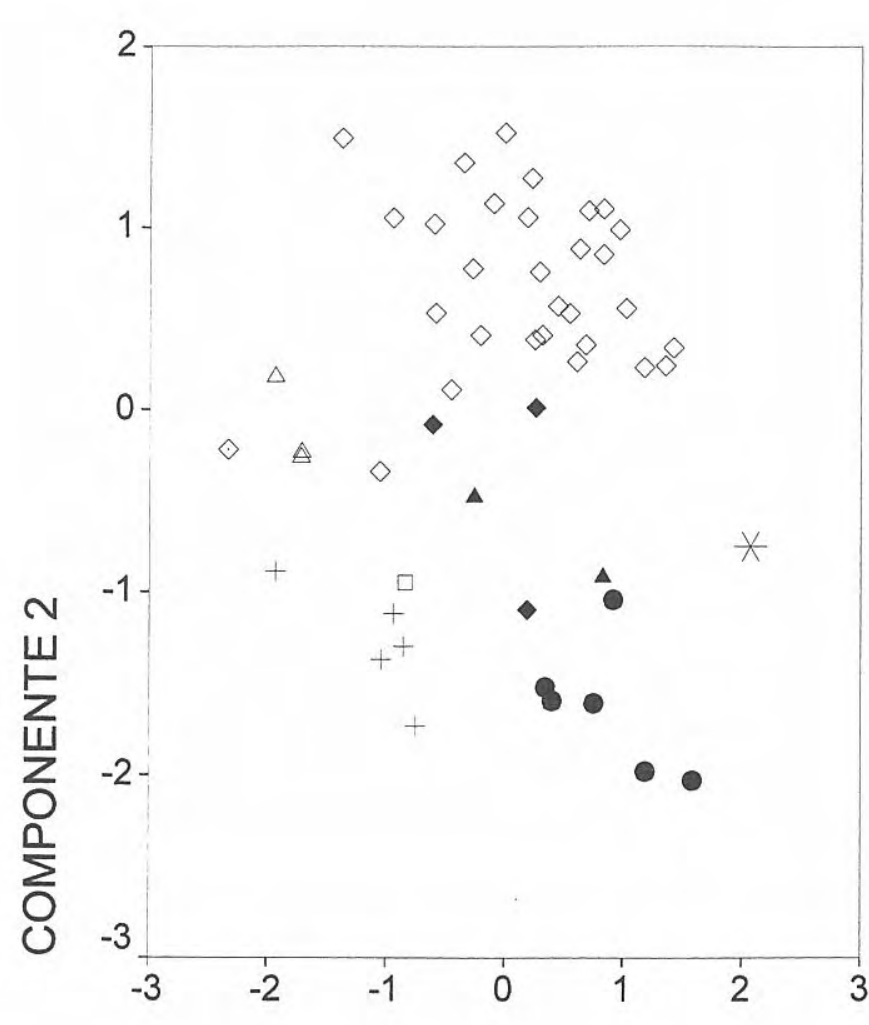

\section{COMPONENTE 1} LOCALIDADES

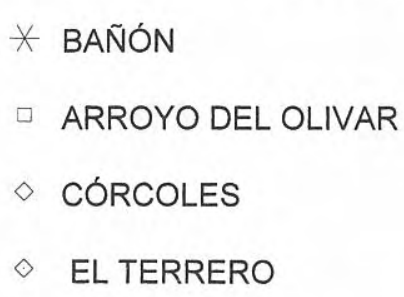

- PARACUELlOS 5

$\triangle \quad$ PTE. VALLECAS

Figura 10. Escafoides de Anchitherium sp. Representación gráfica del componente 1 contra el componente 2. Scaphoid of Anchitherium sp. Scatterplot of Factor 1 and Factor 2.

\author{
ORDEN ARTIODACTYLA Owen, 1848 \\ Superfamilia Suoidea Gray, 1821 \\ Familia Suidae Gray, 1821
}

\section{Suidae indet.}

Fragmento de premolar (BN-s.s.)

El fragmento identificado es tan pequeño que no aporta más datos que la presencia en la fauna de Bañón de una especie de suido. 


\begin{tabular}{|l|cccccc|}
\cline { 2 - 7 } \multicolumn{1}{c|}{} & \multicolumn{7}{c|}{ Componente } \\
\hline & $\mathbf{1}$ & $\mathbf{2}$ & $\mathbf{3}$ & $\mathbf{4}$ & $\mathbf{5}$ & $\mathbf{6}$ \\
\hline Medida 1 & $-0,262$ & 0,918 & $7,58 \mathrm{E}-03$ & $-0,199$ & $7,22 \mathrm{E}-02$ & $-0,21$ \\
Medida 2 & $-0,378$ & 0,864 & 0,193 & 0,175 & $-2,54 \mathrm{E}-03$ & 0,208 \\
Medida 3 & 0,893 & $1,65 \mathrm{E}-02$ & 0,31 & 0,269 & $9,87 \mathrm{E}-02$ & $-0,153$ \\
Medida 4 & 0,873 & 0,261 & $5,77 \mathrm{E}-02$ & $-0,103$ & $-0,395$ & $1,30 \mathrm{E}-02$ \\
Medida 5 & 0,752 & 0,4 & $-0,495$ & 0,149 & $7,88 \mathrm{E}-02$ & $2,27 \mathrm{E}-02$ \\
Medida 6 & 0,912 & $2,29 \mathrm{E}-02$ & 0,132 & $-0,272$ & 0,236 & 0,145 \\
\hline
\end{tabular}

Tabla 8. PCA FC1 de Anchitherium sp. Matriz de componentes. PCA FCl of Anchitherium sp. Factor Score Coefficients.

Pecora indet.

Fig. 3e-f

\section{Astrágalo (B $\tilde{N}-75)$}

Está bastante erosionado. El cóndilo articular lateral de la tróclea proximal es bastante más alto que el medial. La tróclea plantar es una superficie articular muy ancha y convexa, que ocupa prácticamente toda la superficie ventral del hueso, y contacta distalmente con el cóndilo medial de la tróclea distal. El cóndilo lateral de la tróclea distal es un poco más grande que el medial y enlaza suavemente con la garganta central. El cóndilo medial es de perfil recto y enlaza en ángulo, más bruscamente, con la garganta central.

\section{Discusión}

El astrágalo compacto de lados paralelos lo identifica como perteneciente a un pécora. El tamaño circunscribe

\begin{tabular}{|c|ccc|}
\hline Componente & Total & \% Varianza & \% Acumulado \\
\hline 1 & 3,759 & 62,642 & 62,642 \\
2 & 1,406 & 23,44 & 86,082 \\
3 & 0,39 & 6,497 & 92,579 \\
4 & 0,215 & 3,587 & 96,166 \\
5 & 0,154 & 2,572 & 98,738 \\
6 & $7,57 \mathrm{E}-02$ & 1,262 & 100 \\
\hline
\end{tabular}

Tabla 9. PCA FC2 de Anchitherium sp. Varianza total explicada.

PCA FC2 of Anchitherium sp. Total Variance Explained. la identificación taxonómica a los géneros Hispanomeryx o Micromeryx, pero al ser imposible determinar a cuál de los dos, y teniendo en cuenta que pertenecen a familias diferentes (Hispanomerycidae y Moschidae, respectivamente), clasificamos este rumiante como Pecora indet.

Familia Cervidae Gray, 1821

Género Heteroprox Stehlin, 1928

\section{Heteroprox moralesi Azanza, 2000}

Fig. 13

\section{Asta (B̃̃ -11) (medidas en Tabla 14)}

Es un asta s.s. que comprende la bifurcación y las ramas que parten de ella. No presenta roseta y no conserva el pedículo. Es similar al ejemplar MNCNPV-136 de Puente de Vallecas, aunque la rama posterior, en lugar de ser única, se divide en dos desde la base, y en general, el asta es un poco más compleja en su estructura, con mayor número de puntas accesorias. La estructura general es de ramificación basal anteroposterior, pero cada rama principal está a su vez bifurcada, lo que da lugar a la presencia de cuatro ramas, morfología correspondiente con los fragmentos de asta s.s. de Paracuellos 5, más complejos que el holotipo (Azanza, 2000). La rama anterior es de sección más elíptica que la posterior, cuyo borde interno está claramente aplanado, y es más ancho. Asimismo, la rama anterior presenta una segunda bifurcación en el ápice, lo que da lugar a dos pequeñas puntas terminales que están rotas.

No hay ornamentación en la cara interna de las puntas. En la externa presenta estriaciones longitudinales, más marcadas en las ramas posteriores, donde llegan a convertirse en surcos

\begin{tabular}{|l|cccccc|}
\cline { 2 - 7 } \multicolumn{1}{c|}{} & \multicolumn{7}{c|}{ Componente } \\
\hline & $\mathbf{1}$ & $\mathbf{2}$ & $\mathbf{3}$ & $\mathbf{4}$ & $\mathbf{5}$ & $\mathbf{6}$ \\
\hline Medida 1 & 0,673 & 0,677 & $-3,46 \mathrm{E}-02$ & 0,118 & $-2,72 \mathrm{E}-01$ & $2,17 \mathrm{E}-02$ \\
Medida 2 & 0,639 & 0,681 & 0,265 & $1,78 \mathrm{E}-03$ & $2,41 \mathrm{E}-01$ & $2,03 \mathrm{E}-02$ \\
Medida 3 & 0,84 & $-4,89 \mathrm{E}-01$ & $1,32 \mathrm{E}-02$ & 0,102 & $1,74 \mathrm{E}-02$ & 0,212 \\
Medida 4 & 0,888 & $-0,207$ & $1,57 \mathrm{E}-01$ & $-0,367$ & $-9,34 \mathrm{E}-02$ & $-3,25 \mathrm{E}-02$ \\
Medida 5 & 0,842 & $8,76 \mathrm{E}-02$ & $-0,516$ & $-4,14 \mathrm{E}-02$ & $1,15 \mathrm{E}-01$ & $-4,78 \mathrm{E}-02$ \\
Medida 6 & 0,834 & $-4,43 \mathrm{E}-01$ & 0,165 & 0,233 & $1,27 \mathrm{E}-03$ & $-0,163$ \\
\hline
\end{tabular}

Tabla 10. PCA FC2 de Anchitherium sp. Matriz de componentes. PCA FC2 of Anchitherium sp. Factor Score Coefficients. 


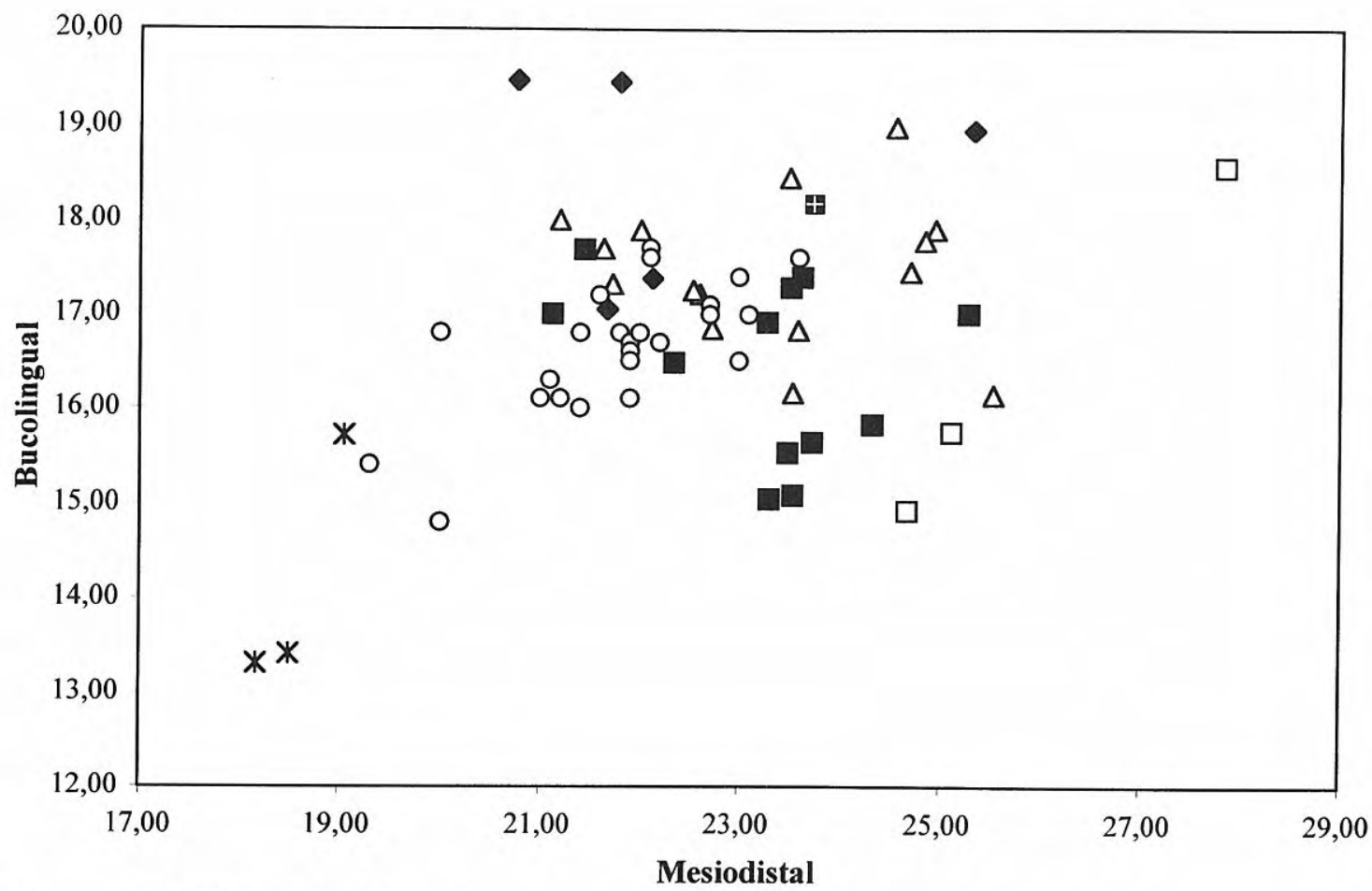
A. alberdiae
A. cursor

$\Delta$ A. matritense

$\square$ A. procerum

* A. aurelianense

O $A$. corcolense

$\rightarrow \mathrm{BAN} O N$

Figura 11. Medidas del p4 de varias especies de Anchitherium, incluida la especie de Bañón. p4 measurements of several Anchitherium species, including the one from Bañón.

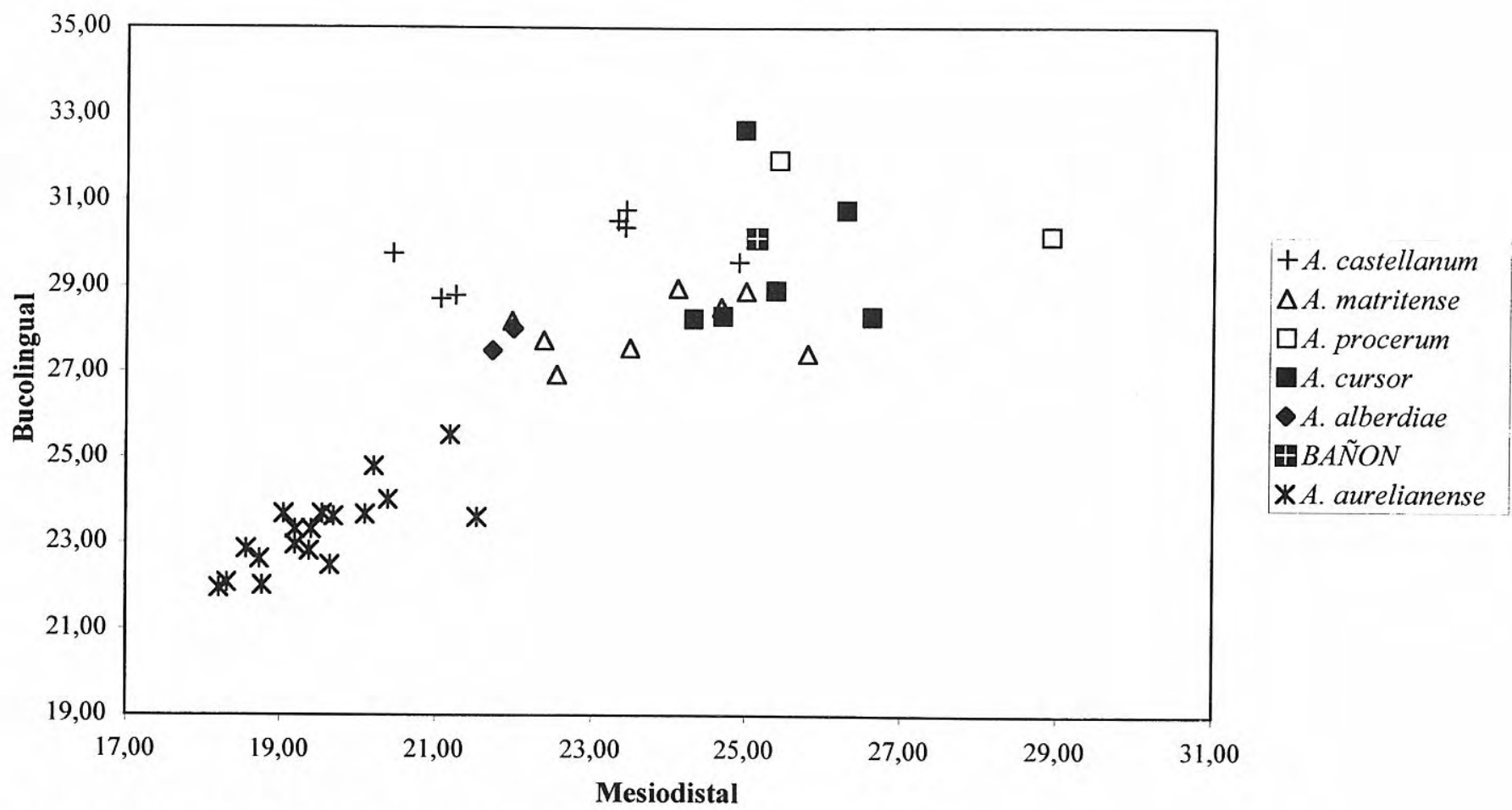

Figura 12. Medidas del P4 de varias especies de Anchitherium, incluida la especie de Bañón. P4 measurements of several Anchitherium species, including the one from Bañon.

bastante profundos, como el que aparece siguiendo el eje longitudinal de la rama principal posterior.

Cabe destacar que además de este ejemplar, se ha encontrado un fragmento de otra asta s. s., perteneciente a la zona basal.

\section{Discusión}

La morfología del asta coincide con las descritas para H. moralesi del Puente de Vallecas, incluyendo la bifurcación dicotómica basal, y la presencia de astas s.s. de morfología compleja que presentan bifurcaciones de $2^{\circ}$ 


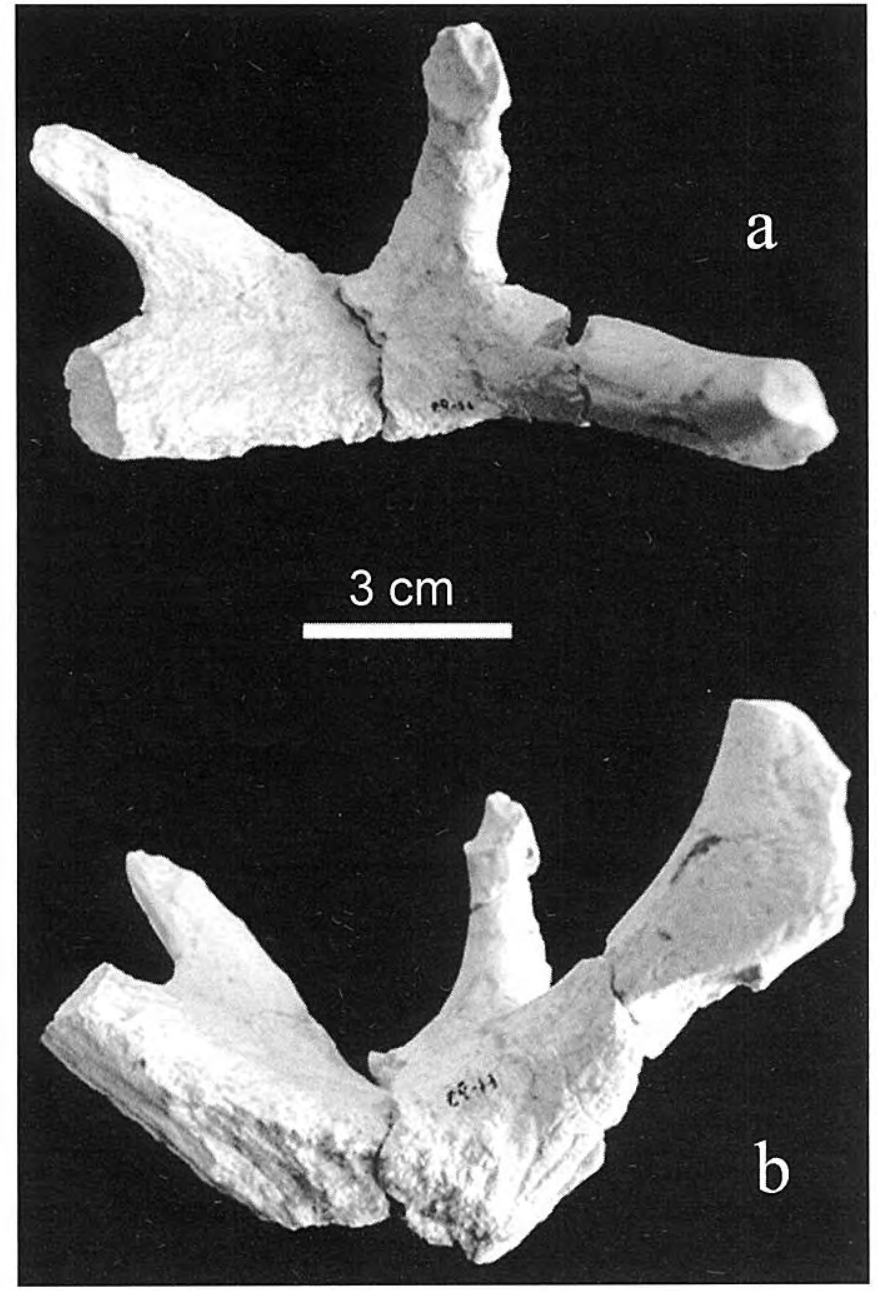

Figura 13. BÑ-11: asta s.s. de Heteroprox moralesi Azanza, 2000 de Bañón.; a, vista dorsal; b, vista medial. BÑ-11: antler s.s. of Heteroprox moralesi Azanza, 2000 from Bañón.; $\boldsymbol{a}$, dorsal view; $\boldsymbol{b}$, medial view.

y $3^{\text {er }}$ orden, acompañadas de aplanamiento y desarrollo de puntas accesorias (Azanza, 2000). Además, hemos realizado un test de la t para comparar las medidas del asta de Bañón con las medidas dadas por Azanza (2000) en la descripción de la especie, y en los casos en que el test se pudo llevar a cabo, debido al bajo número de muestra, no se encontraron diferencias significativas (es decir, p>0,05). Por tanto, clasificamos el cérvido de Bañón como Heteroprox moralesi.

\begin{tabular}{|cccc|}
\hline Componente & Total & \% Varianza & \% Acumulado \\
\hline 1 & 1,973 & 65,761 & 65,71 \\
2 & 0,82 & 27,339 & 93,1 \\
3 & 0,207 & 6,9 & 100 \\
\hline
\end{tabular}

Tabla 11. PCA escafoides de Anchitherium sp. Varianza total explicada.

PCA scaphoid of Anchitherium sp. Total Variance Explained.

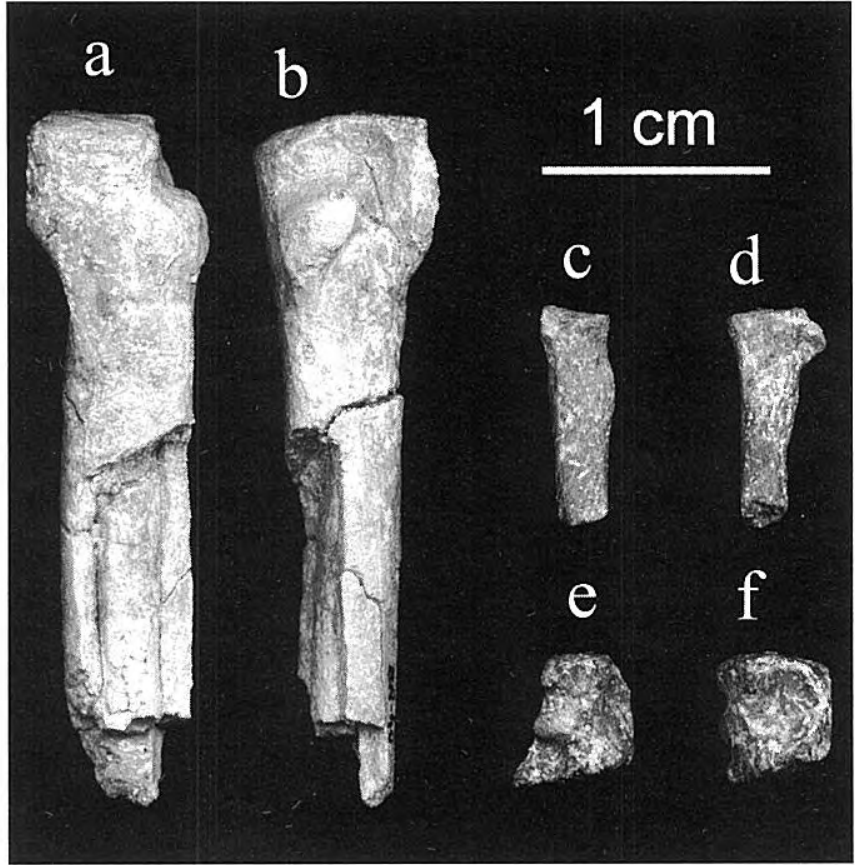

Figura 14. a-b, BÑ-40: fragmento proximal de metatarsiano IV derecho de Hemicyoninae indet. de Bañón; a, vista dorsal; b, vista medial. c-d, BÑ-s. s.: fragmento proximal de metatarsiano II derecho de Mustelidae indet. de Bañón; c, vista dorsal; d, vista medial. e-f, BÑ-63: cuboides derecho de Viverridae indet. de Bañón; e, vista medial; f, vista ventral.

$\boldsymbol{a}-\boldsymbol{b}, B \tilde{N}-40:$ proximal fragment of right metatarsal IV of Hemicyoninae indet. from Bañón; $a$, dorsal view; $b$, medial view. $c-d, B \tilde{N}-$ s. s.: proximal fragment of right metatarsal II of Mustelidae indet. from Bañón; c, dorsal view; $d$, medial view. $\boldsymbol{e}-f, B \tilde{N}-63$ : right cuboid of Viverridae indet. from Bañón; e, medial view; $f$, ventral view.

ORDEN CARNIVORA Bowdich, 1821

Familia Ursidae Fischer, 1817

Subfamilia Hemicyoninae Frick, 1926

Hemicyoninae indet. Fig. 14a-b

Fragmento proximal de Metatarsiano IV derecho (BÑ40) (Tabla 15)

La pieza responde a la morfología típica de este grupo, pero su tamaño es menor que la homóloga de Hemicyon sansaniensis o Plithocyon armagnacensis de Sansan.

La superficie de articulación proximal tiene forma rectangular y se halla ligeramente inclinada en sentido medial. En su cara lateral se observa la profunda depresión que forma parte,junto con su borde proximal, de la superficie de articulación con el metatarsiano V. En la cara medial se observan las facetas de articulación con el metatarsiano III: en el borde anterior se desarrolla una gran faceta, de forma subtriangular, y con su plano articular 


\begin{tabular}{|c|ccc|}
\cline { 2 - 4 } \multicolumn{1}{c|}{} & \multicolumn{3}{c|}{ Componente } \\
\hline \multirow{3}{c}{ Longitud } & $\mathbf{1}$ & $\mathbf{2}$ & $\mathbf{3}$ \\
\cline { 2 - 4 } Anchura & 0,809 & $-0,527$ & 0,261 \\
Altura & 0,66 & 0,734 & 0,159 \\
& 0,94 & $-6,23 \mathrm{E}-02$ & 0,337 \\
\hline
\end{tabular}

Tabla 12. PCA escafoides de Anchitherium sp. Matriz de componentes.

PCA escaphoid of Anchitherium sp. Factor Score Coefficients.

orientado en sentido medial; cerca del borde posterior aparece la otra faceta de articulación, claramente alargada en sentido próximo-distal, y con su plano orientado en sentido postero-medial.

\section{Familia Mustelidae Swainson, 1835}

Mustelidae indet.

Fig. 14c-d

Fragmento proximal de Metatarsiano II derecho (BÑ-s. s.) (medidas en Tabla 15)

La superficie proximal de articulación tiene forma triangular, con una marcada escotadura en el borde lateral y un vértice posterior afilado y rugoso; al contrario que en hiénidos primitivos, vivérridos y félidos, en los que este proceso se inclina en sentido medial, en el ejemplar de Bañón este proceso carece de inclinación y se orienta posteriormente, de manera similar a la de mustélidos como Meles. La cara medial presenta, en su borde anterior, una zona rugosa de forma triangular en la que articula el metatarsiano I; esta zona es algo más amplia que en Meles, pero su morfología es muy similar a la de éste, con el borde posterior crestado y claramente destacado del hueso, diferente del modelo vivérrido-félido en el que el borde posterior es suave y no desarrolla cresta alguna. En la cara lateral, las dos facetas para la articulación con el metatarsiano III y el ectocuneiforme están claramente separadas por una pequeña cresta central, algo más marcada que en Meles. La faceta anterior, cóncava y de forma elipsoidal, no aparece dividida en una zona proximal para el ectocuneiforme y otra distal para el metatarsiano III, como se observa en los félidos, sino que constituye una superficie única, con el borde anterior algo más crestado que en Meles.

\begin{tabular}{|r|ccc|ccc|}
\cline { 2 - 7 } \multicolumn{1}{c|}{} & \multicolumn{3}{c|}{ P4 } & \multicolumn{3}{c|}{ p4 } \\
\cline { 2 - 7 } & $\mathrm{M}$ & $\mathrm{B}$ & $\mathrm{M} / \mathrm{B}$ & $\mathrm{M}$ & $\mathrm{B}$ & $\mathrm{M} / \mathrm{B}$ \\
\hline A. cursor & $\mathrm{N}$ & $\mathrm{N}$ & $\mathrm{N}$ & $\mathrm{N}$ & $\mathrm{N}$ & $\mathrm{N}$ \\
A. alberdiae & $\mathrm{N}$ & $\mathrm{p}=0,05$ & $\mathrm{~N}$ & $\mathrm{~N}$ & $\mathrm{~N}$ & $\mathrm{~N}$ \\
A. castritense & $\mathrm{N}$ & $\mathrm{p}=0,05$ & $\mathrm{~N}$ & $\mathrm{~N}$ & $\mathrm{~N}$ & $\mathrm{~N}$ \\
A. corcolanum & $\mathrm{N}$ & $\mathrm{N}$ & $\mathrm{N}$ & $\mathrm{N}$ & $\mathrm{N}$ & $\mathrm{N}$ \\
\hline
\end{tabular}

Tabla 13. Resultado de las t de Student para la dentición de Anchitherium de Bañón. La N representa la ausencia de diferencias significativas; cuando éstas existen se representa con su p correspondiente.

$t$-Student test results for the dentition of Anchitherium from Bañón. When Bañón material can be included into the distribution of the compared species, there is represented by an $N$; when it is different, it is represented with the correspondent probability.

La faceta posterior, por el contrario, presenta una morfología más similar a la de los félidos que a la de los mustélidos, ya que es ligeramente cóncava y no plana como en estos últimos.

Familia Viverridae Gray, 1821

Viverridae indet. Fig. $14 \mathrm{e}-\mathrm{f}$

Cuboides derecho (BÑ -63) (medidas en Tabla 15)

La superficie de articulación proximal tiene forma rectangular y se halla marcadamente inclinada en sentido lateral, mientras que la superficie de articulación distal es ligeramente cóncava y de forma redondeada; en su borde posterior se observa el gran desarrollo del reborde por cuya superficie distal discurre el tendón del músculo peroneus longus; dicho reborde se proyecta marcadamente en sentido posterior, al igual que en los vivérridos, mientras que en los mustélidos está muy poco desarrollado; en hiénidos y félidos la proyección es más bien postero-lateral. En vista medial se observa la marcada curvatura del borde anterior, cuyo vértice antero-distal se proyecta notablemente en sentido anterior con relación al antero-proximal. La faceta de

\begin{tabular}{|ccc|ccccccc|}
\cline { 2 - 8 } \multicolumn{1}{c|}{} & \multicolumn{2}{c|}{ Tramo basal } & \multicolumn{2}{c|}{ Rama anterior } & \multicolumn{2}{c|}{ Rama posterior } & \multicolumn{2}{c|}{ Rama supernumeraria } \\
\hline Yacimientos & DAPd & DTd & DAPb & DTb & DAPb & DTb & DAPb & DTb \\
\hline BAÑ́N & 30,7 & 21,3 & - & 15,7 & 15,5 & 11,8 & 13,9 & 12,5 \\
MNCNM/CR-3054 & 28,2 & 21,4 & - & - & 14,4 & 25,6 & - & - \\
MNCNM/PV-136 & 32,38 & - & 17,6 & 18 & 17,8 & 18,2 & 11,7 & 12,2 \\
MNCNM/CR-3216 & - & - & - & - & 33 & 27 & 15,8 & 13,6 \\
MNCNM/CR-3214 & 23,9 & 20,9 & - & - & 28,2 & 18,3 & 15 & 11,9 \\
MNCNM/PV-138 & - & - & 22,2 & 18,8 & - & - & - & - \\
\hline
\end{tabular}

Tabla 14. Medidas del asta de Bañón, comparadas con las de Puente de Vallecas (tomado de Azanza, 2000). Antler measurements from Bañón, compared with the Puente de Vallecas sample (from Azanza, 2000). 


\begin{tabular}{|ccccc|}
\hline Sigla & Pieza & Taxón & Longitud antero-posterior & Anchura latero-medial \\
\hline BÑ-40 & Metatarsiano IV & Hemicyoninae indet. & 15,91 & 10,78 \\
BÑ-s. s. & Metatarsiano II & Mustelidae indet. & 8,29 & 5,82 \\
\hline
\end{tabular}

\begin{tabular}{|cccccc|}
\hline Sigla & Pieza & Taxón & Altura próximo-distal & $\begin{array}{c}\text { Anchura latero-medial } \\
\text { Superficie proximal }\end{array}$ & $\begin{array}{c}\text { Longitud antero-posterior } \\
\text { Superficie proximal }\end{array}$ \\
\hline BÑ- 63 & Cuboides & Viverridae indet. & 11,54 & 9,59 & 5,66 \\
\hline
\end{tabular}

Tabla 15. Medidas de los restos postcraneales de los carnívoros de Bañón. Measurements of the postcranial bones of Bañon carnivores.

articulación con el ectocuneiforme sólo ocupa la mitad anterior de la zona central de esta cara, al contrario que en félidos, hiénidos y mustélidos en los que se prolonga en sentido postero-distal.

\section{Discusión}

Los restos de carnívoros de Bañón son escasos, y pertenecen a elementos postcraneales, por lo que sólo han podido ser identificados a nivel de familia. Sin embargo esta fauna puede enmarcarse claramente entre las típicas durante el Aragoniense medio y superior, en las cuales hemicioninos, mustélidos y vivérridos eran grupos ampliamente distribuidos y bien conocidos.

\section{CONCLUSIÓN}

Los restos de macromamíferos de los nuevos yacimientos de Bañón y Balsete son escasos y se encuentran en un pobre estado de conservación; sus faunas pueden encuadrarse dentro de las ya conocidas para el Aragoniense medio (Balsete) y superior (Bañón).

El yacimiento de Balsete ha podido ser datado debido a la presencia de Hispanotherium matritense, que en la Península Ibérica caracteriza la biozona D del Aragoniense medio (MN 5), edad que proponemos para el nuevo yacimiento.

La relación de fauna recuperada del yacimiento de Bañón es la siguiente:

Ave indet.

Microfauna

Gomphotheriidae indet.

Rhinocerotidae indet.

Anchitherium sp.

Suidae indet.

Pecora indet.

Heteroprox moralesi

Hemicyoninae indet.

Viverridae indet.

Mustelidae indet.

La presencia del cérvido Heteroprox moralesi, conocido en España unicamente en la zona MN 6 del Aragoniense, ha permitido precisar la edad del yacimiento. La nueva cita de Heteroprox moralesi amplía su registro paleogeográfico, ya que esta especie sólo había sido citada en la cuenca de Madrid, aunque se conocía H. larteti en Arroyo de Val (Zaragoza) (Azanza, 2000).

\section{AGRADECIMIENTOS}

Al Dr. Enrique Sanz, por la elaboración de los aspectos geológicos del proyecto. Al Dr. Jan van der Made, que nos proporcionó datos métricos sobre restos de cérvidos. A Susana Fraile y al Dr. Jorge Morales, del MNCN de Madrid, por cedernos material todavía en estudio, para realizar algunas de las comparaciones. A Soledad Cuezva, Javier Élez e Israel Polonio, que colaboraron activamente con nosotros. Queremos agradecer también al servicio de Patrimonio Arqueológico, Paleontológico y de Parques Culturales, de la Dirección General de Aragón las facilidades dadas en todo momento para la realización de este trabajo.

Este estudio se ha llevado a cabo en el contexto del proyecto D. G. E. S. PB-98-0503 titulado "Cambios mayores y ciclicidad sedimentaria en las secuencias neógenas de precipitación salina de las cuencas de Montalbán y Calatayud: geomarcadores de la evolución paleoclimática en cuencas terciárias ibéricas".

\section{BIBLIOGRAFÍA}

Alférez, F., Íñigo, C., Molero, G. y Maldonado, E. 1999. El registro fósil de Córcoles (Guadalajara), España: reflejo de la vida en La Alcarria en el Mioceno inferior. In: $L a$ Huella del Pasado: Fósiles de Castilla-La Mancha (Coords. E. Aguirre e I. Rábano). Patrimonio Histórico. Arqueología, 16. Comunidad de Castilla La Mancha, 261-274.

Astibia, H. 1987. Los macromamíferos del Mioceno medio de Tarazona de Aragón (Depresión del Ebro, provincia de Zaragoza). Paleontologia i Evolució, 21, 11-42.

Astibia, H. y Mazo, A. V. 1988. Los mastodontes (Proboscidea, Mammalia) del Mioceno medio de Tarazona de Aragón (Zaragoza, España). Estudios Geológicos, 44, 329-338. 
Azanza, B. 2000. Los Cervidae (Artiodactyla, Mammalia) del Mioceno de las Cuencas del Duero, Tajo, CalatayudTeruel y Levante. Memorias del Museo Paleontológico de la Universidad de Zaragoza, 8, 376 pp.

Casas, A. M., Casas, A., Pérez, A., Tena, S., Barrier, L., Gapais, D. and Nalpas, T. 2000. Syntectonic sedimentation and thrust-and-fold kinematics at the ultra-mountain Montalban Basin, Spain. Geodinamica Acta, 1, 1-17.

Cerdeño, E. 1989. Revisión de la Sistemática de los rinocerontes del Neógeno de España. Tesis doctoral. Facultad de Ciencias Biológicas, Universidad Complutense de Madrid (inédito), 429 pp.

Crusafont, M. y Villalta, J.F. 1947. Sobre un interesante Rinoceronte (Hispanotherium nov. gen.) del Valle del Manzanares. Nota preliminar. Las Ciencias, 22 (4), 869883.

Cuvier, G. 1817. Le regne animal. Déterville, Paris

Daams, R. and Freudenthal, M. 1988. Synopsis of the Dutch-Spanish collaboration program in the Aragonian type area, 1975-1986. Scripta Geologica, Special Issue, 1, 3-18.

Daams, R. and van der Meulen, A. J. 1992. Paleoenvironmental and Paleoclimatic interpretation of micromammal faunal successions in the upper Oligocene and Miocene of North central Spain. Paléobiologie continentale, Montpellier, 14, 241-257.

Eisenmann, V., Alberdi, M. T., De Giuli, C. and Staesche, U. 1988. Collected papers after the New York International Hipparion Conference, 1981. In: Studying Fossil Horses. Vol. 1: Methodology (Eds. M. Woodburne and P. Sondaar). E. J. Brill, 1-71.

Ginsburg, L. 1961. La Faune des Carnivores Miocènes de Sansan (Gers). Mémoires du Museum National d'Histoire Naturelle, serie C, Sciences de la Terre, 9, 1190.

Ginsburg, L. et Telles Antunes, M. T. 1979. Les Rhinocérotidés du Miocène inferieur et moyen de Lisbonne (Portugal). Sucession stratigraphique et incidences paleogeographiques. Comptes Rendus de l'Academie des Sciences de Paris, 288, 493-495.

Heissig, K. 1974. Neue Elasmotherini (Rhinocerotidae, Mammalia) aus dem Obermiozän Anatoliens. Mitteilungen der Bayerischen Staatssammlung fur
Paläeontologie und historische Geologie, 14, 21-35.

Heissig, K. 1976. Rhinocerotidae (Mammalia) aus der Anchitherium-fauna Anatoliens. Geologisches Jahrbuch, 19, 1-121.

Hernández Pacheco, F. y Crusafont, M. 1960. Primera caracterización paleontológica del Terciario de Extremadura. Boletín de la Real Sociedad Española de Historia Natural, 68 (2), 275-282.

Mazo, A. V. 1997. Revisión de los mastodontes de España. Tesis doctoral. Universidad Complutense de Madrid (inédita), $419 \mathrm{pp}$.

Meyer, H. von. 1834. Die fossilen Zähne und Knochen undihre Ablagerung in der Gegend von Georgesmünd in Bayern. Frankfurt am Main, Verlag v. J.D. Sauerländer.

Morales, J., Alcalá, L., Hoyos, M., Montoya, P., Nieto, M., Pérez, B. y Soria, D. 1993. El yacimiento del Aragoniense medio de La Retama (Depresión Intermedia, Provincia de Cuenca, España): significado de las faunas con Hispanotherium. Scripta Geologica, 103, 23-39.

Prado, C. de 1864. Descripción física y geológica de la provincia de Madrid. Junta General de Estadística, 219 pp.

Sánchez, I. M., Salesa, M. J. y Morales, J. 1998. Revisión sistemática del género Anchitherium Meyer, 1834 (Equidae; Perissodactyla) en España. Estudios Geológicos, 54, 39-63.

Sanz-Rubio, E. 1999. Análisis de los sistemas deposicionales carbonatados y evaporíticos del Neógeno de la cuenca de Calatayud (provincia de Zaragoza). Tesis doctoral. Facultad de Ciencias Geólogicas, Universidad Complutense de Madrid (inédita), 579 pp.

Soria, D., Amezua, L., Daams, R., Fraile, S., Herráez, E., Morales, J., Nieto, M., Peláez-Campomanes, P., Salesa, M. J. y Sánchez, I. M. 2000. Faunas del Mioceno. In: Patrimonio Paleontológico de la Comunidad de Madrid (Coords. J. Morales, M. Nieto, L. Amezua, S. Fraile, E. Gómez, E. Herráez, P. Peláez-Campomanes, M. J. Salesa, I. M. Sánchez y D. Soria). Serie "Arqueología, Paleontología y Etnografía”. Vol. 6. Comunidad Autónoma de Madrid, 110-129.

Stehlin, H. G. 1928. Uber die systematische Stellung des Genus Leptobos. Eclogae Geologicae Helvetica, 21 (1), 217-218. 